\title{
Effects of fourth-order fiber dispersion on ultrashort parabolic optical pulses in the normal dispersion
}

\author{
regime
}

\author{
Brandon G. Bale, ${ }^{1}$ Sonia Boscolo, ${ }^{1}$ Kamal Hammani, ${ }^{2}$ and Christophe Finot ${ }^{2}$ \\ ${ }^{1}$ Photonics Research Group, School of Engineering and Applied Science, Aston University, \\ Birmingham B4 7ET, UK \\ ${ }^{2}$ Laboratoire Interdisciplinaire Carnot de Bourgogne, UMR 5209 CNRS-Université de \\ Bourgogne, 21078 Dijon, France
}

\begin{abstract}
We propose a new method for the generation of both triangular-shaped optical pulses and flat-top, coherent supercontinuum spectra using the effect of fourthorder dispersion on parabolic pulses in a passive, normally dispersive highly nonlinear fiber. The pulse re-shaping process is described qualitatively and is compared to numerical simulations.
\end{abstract}

OCIS codes: $140.4050,060.4230$.

\section{Introduction}

Optical pulses with a parabolic intensity profile and a linear frequency chirp have generated a great deal of attention in the last decade [1]. Parabolic pulses propagate in optical fibers with normal second-order (group-velocity) dispersion (SOD) in a self-similar manner, holding certain relations (scaling) between pulse power, duration and chirp parameter, and can tolerate strong nonlinearity without wave breaking [2]. In the presence of linear amplification, these solutions, so-called "similaritons", possess the remarkable property of being a global 
attractor to the system for arbitrary initial conditions [3-5]. Experimental demonstrations relying on amplification from either rare-earth doping [3,6] or Raman scattering [7] have been achieved. The unique properties of parabolic pulses have stimulated numerous applications. Experimental interest has been strongly driven by the possibility of fully cancelling the linear chirp and thus generating ultrashort high-power pulses with very low substructures $[3,6,8]$. Moreover, recent fiber lasers which use self-similar pulse shaping in the normal dispersion regime have been demonstrated to achieve high-energy pulses [9-11]. Parabolic pulses have also enabled new techniques or noticeable improvements of existing techniques of ultrafast all-optical signal processing for high-bit rate telecommunications based on the quasi-instantaneous Kerr response of highly nonlinear optical fibers [12-16].

Various techniques for the precise synthesis, control and manipulation of the temporal shape of optical pulses have become increasingly important for many scientific applications, including amongst others, ultrahigh-speed optical telecommunications and nonlinear optics. For instance, various transform-limited pulse shapes were generated by temporal coherent synthesis using a multi-arm interferometer [17]. Further, the generation of pulses with flattop [18], parabolic $[8,15]$ and sawtooth (asymmetric triangular) [19] shapes has been demonstrated using superstructured fiber Bragg grating technology. Another alternative to achieve the desired temporal optical pulse shaping is to use nonlinear effects in optical fibers. In addition to fiber amplifiers and lasers, parabolic pulses can be generated in passive fibers provided a suitable longitudinal variation of the dispersion is introduced [20,21]. Recently, a simple approach to the generation of parabolic pulses that uses progressive nonlinear pulse re-shaping and subsequent pulse stabilization in two carefully chosen normally dispersive (ND) fiber segments has been demonstrated [22]. Further, the combination of pulse prechirping and nonlinear propagation in a section of ND fiber has been introduced [23] and demonstrated [24] as a method for passive nonlinear pulse shaping, which provides a simple way of generating various temporal waveforms including triangular profiles. The simple intensity profile of triangular pulses is highly desired for a range of photonic applications. 
Sawtooth pulses have been shown to enhance the performance of wavelength converters based on self-phase modulation (SPM) in fiber and offset filtering [19]. Time-domain adddrop multiplexing based on cross-phase-modulation (XPM)-induced frequency shifting using saw tooth control pulses was examined [25]. Novel techniques using XPM with triangular pump pulses in a nonlinear Kerr medium were introduced to achieve the doubling of optical pulses in both the frequency and time domains [26] as well as to realize time-to-frequency mapping of multiplexed signals in high-speed fiber communication systems [27].

One of the most well known examples of pulse re-shaping in the spectral domain is in supercontinuum (SC) generation $[28,29]$. Typically the broadest spectra are generated when pumping occurs in the anomalous dispersion regime of the fiber, where the broadening mechanism is strongly influenced by soliton dynamics [29]. However, solitonic effects and modulation instability lead to decreased temporal coherence and spectral flatness of the continuum generated in the anomalous dispersion regime, especially when picosecond pump pulses are used [30,31]. Many applications require the conservation of a short pulse in the time domain while generating a flat and coherent SC spectrum. Previous attempts have been made to overcome this drawback by using dispersion-flattened photonic crystal fibers (PCFs) continuously tapered from anomalous to normal dispersion $[32,33]$. However, due to limited availability of such fiber tapers, it is interesting to consider fibers with longitudinally constant dispersion profiles, where a sufficiently broad, stable and flat-top spectrum can still be achieved [34-36]. Such an approach combined with PCFs has recently stimulated experimental attention with several results exhibiting the high coherence and flatness (when plotted on a logarithmic scale) of the resulting supercontinuum.

In this work we qualitatively and numerically show that initially parabolic pulses propagating in a ND fiber with fourth-order dispersion (FOD) can result in temporal triangular intensity profiles and broad, compact, flat spectral profiles. The performance allowed by parabolic pulses is shown to be substantially better compared to more conventional input pulse shapes such as Gaussian or hyperbolic secant pulses. The progressive re-shaping of a 
parabolic pulse driven by the FOD in a ND nonlinear fiber not only provides an alternative passive method for generating triangular-shaped pulses with respect to that introduced in [23], but also allows for remarkably flat spectral intensities at a particular point in the evolution when plotted on a linear scale.

\section{Qualitative Analysis}

Our efforts are motivated by the progress of all-normal dispersion PCFs with flattened dispersion characteristics. Indeed, recent theoretical and experimental works have reported the design and use of fibers with all-normal convex dispersion profiles [35-38]. Specifically, we will base our discussion on the fiber properties demonstrated in [37] with a dispersion profile as shown in Fig. 1 and pumping occurring at the extremum of dispersion.

The flattened dispersion characteristics around the pump wavelength are critical in that the effect of third-order dispersion, which can degrade the self-similar parabolic pulse propagation [39], is significantly reduced below that of FOD. Thus we model pulse propagation by the normalized generalized nonlinear Schrödinger equation [40]

$$
i u_{\xi}-\frac{1}{2} u_{\tau \tau}+N^{2}|u|^{2} u=-\frac{\delta}{24} u_{\tau \tau \tau \tau}
$$

where the dimensionless quantities $u(\xi, \tau)=U / \sqrt{P_{0}}, \xi=z / L_{D}$ and $\tau=t / T$ are introduced. Here, $U(z, t)$ is the electric field envelope in the co-moving system of coordinates, $T$ and $P_{0}$ are the characteristic temporal pulse width and the peak power of the initial pulse, respectively, and $L_{D}=T^{2} / \beta_{2}$ is the dispersion length, with $\beta_{2}$ being the SOD coefficient. The two normalized parameters in Eq. (1) are the energy parameter $N^{2}=L_{D} / L_{N L}$, where $L_{N L}=$ $1 /\left(\gamma P_{0}\right)$ is the nonlinear length, and the effective FOD strength parameter $\delta=\beta_{4} /\left(\beta_{2} T^{2}\right)$. The parameters $\beta_{4}$ and $\gamma$ are the fiber FOD and Kerr nonlinearity coefficients, respectively. Although microstructured fibers has now enabled FOD coefficients where $\beta_{4}<0$, in this work we will restrict our analysis to positive FOD coefficients $(\delta>0)$. Indeed high-power picosecond pulses propagating in a PCF with negative FOD coefficient $(\delta<0)$ experience 
severe pulse degradation arising from fourth-order scalar modulation instability [41], which may ultimately lead to the splitting of the pulse into ultrashort solitonic substructures [42].

To get a qualitative understanding of the re-shaping process, we do a standard decomposition by assuming $u(\xi, \tau)=\sqrt{P(\xi, \tau)} \times e^{i \phi(\xi, \tau)}$ in Eq.(1), giving

$$
\begin{aligned}
\phi_{\xi} & =-\frac{1}{2}\left[\frac{\sqrt{P}_{\tau \tau}}{\sqrt{P}}-\Omega^{2}\right]+N^{2} P+\frac{\delta}{24} R_{1} \\
P_{\xi} & =[P \Omega]_{\tau}-\frac{\delta}{12} R_{2}
\end{aligned}
$$

where $\Omega \equiv \phi_{\tau}$ is the chirp of the pulse and the FOD contributions are

$$
\begin{aligned}
& R_{1}=\frac{1}{\sqrt{P}}\left(\sqrt{P}_{\tau \tau \tau \tau}-6\left[\sqrt{P}_{\tau} \Omega^{2}\right]_{\tau}\right)+\Omega^{4}-3 \Omega_{\tau}^{2}-4 \Omega \Omega_{\tau \tau} \\
& R_{2}=2 \sqrt{P}\left(2\left[\sqrt{P}_{\tau \tau} \Omega\right]_{\tau}+\left[\sqrt{P}_{\tau} \Omega_{\tau}\right]_{\tau}\right)-\left[P\left(2 \Omega^{3}-\Omega_{\tau \tau}\right)\right]_{\tau} .
\end{aligned}
$$

For initial high intensity $\left(N^{2} \gg 1\right)$ linear chirped parabolic pulses where $P(0, \tau)=$ $\left(1-\tau^{2}\right) \theta(1-|\tau|)$ and $\Omega(0, \tau)=2 C_{0} \tau$ with $\theta(x)$ being the Heaviside function and $C_{0}$ the (normalized) chirp coefficient, the magnitude (in some norm) of each term in Eqs. (2-3) can be analyzed. We find numerically that only certain terms contribute to the main pulse shaping process. Note that we consider $C_{0}<0$, thus neglecting the transient stage of spectral compression if $C_{0}>0$ [43]. Taking the leading order gives an approximate coupled system for the dynamics

$$
\begin{aligned}
\phi_{\xi} & =\frac{1}{2} \Omega^{2}\left(1+\frac{\delta}{12} \Omega^{2}\right)+N^{2} P \\
P_{\xi} & =\Omega\left(1+\frac{\delta}{6} \Omega^{2}\right) P_{\tau}+\Omega_{\tau}\left(1+\frac{\delta}{2} \Omega^{2}\right) P .
\end{aligned}
$$

For small propagation distances from $\xi=0$ the chirp profile is approximately given from Eq. (4a)

$$
\Omega(\xi, \tau) \sim 2 C_{0} \tau-2 N^{2} \tau\left(1-\frac{2 C_{0}^{2}}{N^{2}}\left(1+\delta C_{0}^{2} \tau^{2}\right)\right) \xi
$$


Since $\delta / N^{2} \ll 1$, the chirp maintains a nearly linear profile in time, and initial wave-breaking is avoided since the chirp is still monotonous. This nearly linear chirp is coupled to the power evolution in Eq. (4b) and leads to a re-shaping process due to the FOD terms. Specifically, Eq. (4b) is a partial differential equation where the power $P$ has the characteristic velocity [44] and power evolution given by

$$
\begin{aligned}
\frac{d \tau}{d \xi} & =-\Omega\left(1+\frac{\delta}{6} \Omega^{2}\right) \\
\frac{d P}{d \xi} & =\Omega_{\tau}\left(1+\frac{\delta}{2} \Omega^{2}\right) P
\end{aligned}
$$

Neglecting the $\delta / N^{2}$ term in Eq.(5) gives $\Omega \sim \Omega_{0} \tau$, where $\Omega_{0}=2 C_{0}-2 N^{2} \xi_{0}\left(1-2 C_{0}^{2} / N^{2}\right)$ (with $\left.\xi_{0} \ll 1\right)$. Without FOD $(\delta=0)$, parabolic self-similar solutions to Eqs. (4) have been found [2]. In this limit Eq. (6) has solutions $\xi(\tau)=-\log \left(\tau / \tau_{0}\right) / \Omega_{0}$ and $P\left(\xi, \tau_{0}\right)=$ $P\left(0, \tau_{0}\right) \times \exp \left(\Omega_{0} \xi\right)$. For relevant initial conditions, $C_{0}^{2} \ll N^{2}$ giving $\Omega_{0}<0$ so that the power $P\left(\xi, \tau_{0}\right)$ exponentially decays along each characteristic curve. Including FOD in Eq. (6) we find the solutions

$$
\begin{aligned}
\xi\left(\tau, \tau_{0}\right) & =-\frac{1}{\Omega_{0}} \log \left(\frac{k \tau}{\sqrt{1+\frac{\delta}{6} \Omega_{0}^{2} \tau^{2}}}\right) \\
P\left(\xi, \tau_{0}\right) & =P\left(0, \tau_{0}\right) e^{\Omega_{0} \xi} \times R\left(\xi, \tau_{0}\right),
\end{aligned}
$$

where

$$
R\left(\xi, \tau_{0}\right)=\left[\frac{\frac{\delta}{6} \Omega_{0}^{2}-k^{2} e^{2 \Omega_{0} \xi}}{\frac{\delta}{6} \Omega_{0}^{2}-k^{2}}\right]^{3 / 2} e^{-3 \Omega_{0} \xi}
$$

and $k^{2}=1 / \tau_{0}^{2}+\delta \Omega_{0}^{2} / 6$. Figure 2 shows an example of the characteristic curves with and without FOD. With FOD the curves deflect away (towards $\tau= \pm \infty$ ) from those without FOD. Note that that this deflection is more extreme in the wings of the pulse for short propagation distances, leading to a stretching of the pulse (see grey line and dots in Fig. 2). Further, Eq. (7b) shows that the exponential decay of the power along each characteristic curve is multiplied by a functional factor $R\left(\xi, \tau_{0}\right)$ (Eq. (8)) which is shown in Fig. 3 for 
various $\tau_{0}$ values. Since $R(\xi)<1$ for $\left|\tau_{0}\right|>0$ the pulse wings undergo an enhanced power reduction when compared to the evolution without FOD. Thus the overall temporal effect of FOD on linearly chirped parabolic pulse propagation is to stretch and enhance the power reduction in the pulse wings, leading to a triangular profile.

In the spectral domain, once the pulse has been stretched towards a triangular shape, the spectral power evolves towards an M-shape due to the SPM effect $\left(N^{2} \gg 1\right)$. Indeed, for a triangular pulse where $P\left(\xi_{0}, \tau\right) \sim\left(1-\left|\tau / \tau_{T}\right|\right) \theta\left(\tau_{T}-|\tau|\right)$, the nonlinear phase shift acquired upon propagation $\phi(\tau)=N^{2} P\left(\xi_{0}, \tau\right)\left(\xi-\xi_{0}\right)$ leads to a constant, distinct (opposite sign) chirp induced onto the leading (down-shfited) and trailing (up-shifted) edges of the pulse. As a result of this, the outer parts of the pulse spectrum are enhanced, whereas the central parts are suppressed and, thus, the spectrum develops an M-shaped structure. An important point is that in the transition from the initially rounded-top (parabolic) spectral power to an

M-shaped spectral power, the spectral density becomes flat. This flattened spectrum could be useful in SC generation applications. In the following section, we emphasis the qualitative findings through numerical simulations.

\section{Numerical Simulations}

In this section Eq. (1) will be numerically integrated using the standard split-step Fourier method. Following Ref. [37] (see also Fig. 1), the fiber parameters are given by $\beta_{2}=2.5 \times$ $10^{-3} \mathrm{ps}^{2} \mathrm{~m}^{-1}, \beta_{4}=2.0 \times 10^{-7} \mathrm{ps}^{4} \mathrm{~m}^{-1}$ and $\gamma=40 \times 10^{-3}(\mathrm{Wm})^{-1}$. To avoid numerical artifacts due to the unphysical compact nature of the parabolic pulse, we use here slight temporal apodization of the intitial condition based on a fifth-order Gaussian shape with a full-width at half-maximum (FWHM) of $1.6 T$ [8]. The input pulse parameters we use correspond to typical parabolic pulses output from a ytterbium-doped fiber amplifier [3]. They have energy $12 \mathrm{~nJ}$, FWHM pulse duration $2.6 \mathrm{ps}$ and chirp coefficient $-3.35 \pi \mathrm{THz}^{2}$. Both the fiber and initial pulse parameters gives the dispersion length $L_{D}=1.3520 \times 10^{3} \mathrm{~m}$, energy parameter $N^{2}=2.6474 \times 10^{5}$, and FOD parameter $\delta=2.3669 \times 10^{-5}$. Propagation in 
the fiber is considered up to a maximum length of $4 L_{D} \times 10^{-3}$ which is on the order of meters in physical units $\left(\sim 1000\right.$ nonlinear lengths). Note that in this parameter regime $N^{2} \gg 1$, so that $\Omega_{0} \sim-2 N^{2} \xi$. Thus we would expect that increasing or decreasing the initial chirp values will not have a significant effect in the re-shaping process.

During propagation in the fiber, the initial pulse will experience significant re-shaping, evolving towards a triangular profile in the time domain. Different approaches are possible to characterize the pulse shape $[22,23,45]$. Here we choose the parameter of misfit $M_{S}$ between the pulse intensity profile and a parabolic or triangular fit of the same energy and FWHM duration:

$$
M_{S}^{2}=\frac{\int d t\left(P-P_{S}\right)^{2}}{\int d t P^{2}}, \quad S=p, T
$$

where $P_{p}(\tau)$ and $P_{T}(\tau)$ correspond to the respective intensity profiles $P_{p}(\tau)=p_{p}(1-$ $\left.\tau^{2} / \tau_{p}^{2}\right) \theta\left(\tau_{p}-|\tau|\right)$ and $P_{T}(\tau)=p_{T}\left(1-\left|\tau / \tau_{T}\right|\right) \theta\left(\tau_{T}-|\tau|\right)$ with $p_{S}$ being the pulse peak power. To examine the impact of FOD on the evolution of the initial parabolic pulse, we compare the evolution obtained with and without FOD. An example of the longitudinal temporal and spectral pulse evolutions is shown in Fig 4-5. In the absence of FOD, the pulse evolves self-similarly maintaining its parabolic shape both in the time and frequency domains, while undergoing temporal and spectral broadening. Due to the high $N^{2}$ value, these results are consistent with previously found results [2]. In the presence of FOD, significant temporal and spectral re-shaping of the parabolic pulse occurs over short propagation distances. In the temporal domain the wings of the pulse are stretched, generating a triangular shaped pulse as predicted in the previous section. This shape is maintained over a non-negligible propagation distance. In the spectral domain, the power goes through a re-shaping leading to a spectrum with rapidly falling edges and a ripple-free top over a $\Delta \omega \simeq 578$ span (corresponding to approximately $50 \mathrm{THz}$ ) at propagation distance $\xi=0.0011$. The flat-top spectrum is a transient state and for further propagation distance the spectral power evolves into an M-shape due to the triangular nature of the temporal intensity profile along with SPM. As to be expected, the spectral bandwidth approaches a limit as the pulse undergoes 
enhanced temporal broadening and faster peak power reduction [46].

The trends shown in Figs. 4-5 are confirmed in Fig. 6, where the misfit parameter (9) has been calculated over the propagation distance. In the presence of FOD, the misfit parameter to a triangular temporal shape reaches a minimum of $M_{T, o p t} \simeq 0.057$ at the distance $\xi_{\text {opt }}=$ 0.0028, while being below 0.07 over the range $\xi=0.0021$ to the maximum propagation distance considered. Stabilization of the root-mean-square (RMS) pulse spectral bandwidth after the M-shaping of the spectrum has occurred is also evident from Fig. 6. The RMS spectral bandwidth is calculated as $\omega_{R M S}=\left[\int d \omega \omega^{2}|\tilde{u}|^{2} / \int d \omega|\tilde{u}|^{2}\right]^{1 / 2}$, where $\tilde{u}$ is the Fourier transform of the field. The two red dots in Fig. 6 symbolize the points in propagation where (Fig. 6a) the temporal profile is most triangular (shown in inset of Fig. 2(b)) and (Fig. 6b) the spectral profile is most flat with a ripple-free top (shown as the green curve in Fig. 3(b)). Note that typically the best triangular shapes are obtained at larger propagation distances than those where the spectrum is maximally flat. Further, the spectrum generated in presence of FOD saturates earlier and exhibits a lower spectral broadening than the spectrum recorded without FOD, which is a direct consequence of the increased broadening experienced when FOD is taken into account.

\section{A. Impact of FOD and energy parameter}

As described in Section 2, the overall effect of FOD depends on a term whose coefficient is $\delta \Omega_{0}^{2} / 6$, where $\Omega_{0}^{2} \sim 4\left(N^{2}\right)^{2} \xi_{0}^{2}$ (see Eq. (7)). Thus we expect that increasing either the FOD strength parameter $\delta$ (by increasing the physical FOD $\beta_{4}$ ) or the energy parameter $N^{2}$ (by increasing the input pulse peak power) will generate a stronger stretching and enhanced power reduction in the wings of the pulse. Indeed, this will effectively lead to pulse re-shaping for shorter propagation distances as well as being able to obtain an improved triangular shape due to a sharpening of the pulse top. This qualitative description is confirmed by numerical simulations. The influence of the FOD (energy) parameter on the temporal evolution can be seen in Fig. 7a(b) where the longitudinal evolutions of the misfit parameter to a triangular 
temporal shape are shown. For increasing values of $\delta$ or $N^{2}, \xi_{\text {opt }}$ and $M_{T}\left(\xi_{\text {opt }}\right)$ decrease showing that the re-shaping process happens at shorter propagation distances and that more ideal triangular pulse shapes are obtained. In the spectral domain, larger $\delta$ and $N^{2}$ leads to flattening and subsequent M-shaping of the spectral power earlier in propagation distance, as to be expected. The spectrum settles to a smaller bandwidth at large distances for increased $\delta$ where larger $N^{2}$ leads to more spectral broadening and larger bandwidths. Figure 8 illustrates the temporal and spectral power profiles at two significant propagation distances where there is lowest misfit parameter $M_{T}$ (dots in Fig. $7(\mathrm{~b})$ ) and maximally flat spectrum.

\section{B. Impact of initial pulse shape}

We have investigated the effect of FOD on the evolution of an initially linearly chirped parabolic pulse in a ND fiber. Since in our parameter regime $N^{2} \gg 1$, we would expect from Eq. (4a) the the initial pulse shape will have a significant effect on propagation. We consider the evolution of both transform-limited Gaussian pulses $u(0, \tau) \sim \exp \left(-\tau^{2} /\left(2 \tau_{G}^{2}\right)\right)$ and a hyperbolic-secant pulses $u(0, \tau) \sim \operatorname{sech}\left(\tau / \tau_{S}\right)$ and compare to the evolution of a chirpfree parabolic pulse. For all initial conditions, the same pulse energy and FWHM temporal duration are used. The spectro-temporal presentation of the pulses obtained at $\xi=0.0013$ in Fig. 9 highlights the distinctly different pulse evolutions. For the initial parabolic pulse, the initial chirp generated is linear (see Eq. (5)). Upon propagation, this chirp deviates from a strictly linear behavior because the pulse wings evolve at higher velocity than the central part of the pulse as a result of the FOD. However, the chirp remains monotonic and the pulse does not undergo wave breaking. For the initial Gaussian and hyperbolic secant pulse shapes the last term on the right hand side in Eq. (4a) induces a non-monotonic chirp which leads to wave-breaking. In this case, triangular pulse formation is not observed for such initial pulses. Figure 10 shows the temporal and spectral profiles at $\xi=0.0013$ for the three different initial conditions. The high oscillations in the spectrum for the initial Gaussian and 
hyperbolic secant are induced by wave-breaking. The specific initial parabolic pulse profile avoids wave-breaking and generates a flattening of the spectral power and triangular pulse formation in the temporal domain.

\section{Conclusion}

In conclusion, we have investigated the propagation of initially parabolic pulses in a ND fiber in the presence of FOD. For the physical parameter regime explored a qualitative understanding of the re-shaping was obtained and characterized in terms of the parameter $\delta \Omega_{0}^{2} \sim \delta N^{4} \xi_{0}^{2}$. The overall effect of the FOD is to stretch and enhance the power reduction in the wings of the initial parabolic pulse, leading to a temporal triangular profile. Further, the spectral power is re-shaped from a rounded top profile into a M-shape. At a certain point

in this re-shaping process, the spectral power has rapidly falling edges and a ripple-free top over a broad bandwidth. Numerical simulations confirm our qualitative understanding and show that increasing the strength of the FOD or the energy parameter leads to pulse re-shaping for shorter propagation distances as well as being able to obtain an improved temporal triangular profile due to a sharpening of the pulse top. Finally, an initial parabolic pulse profile was shown to be necessary to avoid wave-breaking for such re-shaping dynamics to occur. This work could potentially have impact on applications that rely on temporal triangular pulses as well as those requiring the conservation of a short pulse in the time domain while generating a flat and coherent SC spectrum.

\section{Acknowledgements}

The authors would like to acknowledge support from the Engineering and Physical Sciences Research Council (Grant No. EP/FO2956X/1), the British Council (Alliance Grant No. 10.002) and the Region Bourgogne (PARI PHOTCOM), the Agence Nationale de la Recherche (ANR SOFICARS, ANR-07-RIB-013-03) and the Minist ére des Affaires étrangeres et européenne (Alliance grant 22836ZJ). 


\section{References}

1. C. Finot, J. M. Dudley, B. Kibler, D. J. Richardson, and G. Millot, "Optical parabolic pulse generation and applications," IEEE J. Quantum Electron. 45, 1482-1488 (2009).

2. D. Anderson, M. Desaix, M. Karlsson, M. Lisak, and M. L. Quiroga-Teixeiro, "Wavebreaking-free pulses in nonlinear-optical fibers," J. Opt. Soc. Am. B 10, 1185-1190 (1993).

3. M. E. Fermann, V. I. Kruglov, B. C. Thomsen, J. M. Dudley, and J. D. Harvey, "Selfsimilar propagation and amplification of parabolic pulses in optical fibers," Phys. Rev. Lett. 84, 6010-6013 (2000).

4. S. Boscolo, S. K. Turitsyn, V. Y. Novokshenov, and J. H. B. Nijhof, "Self-similar parabolic optical solitary waves," Theor. Math. Phys. 133 1647-1656 (2002).

5. V. I. Kruglov and J. D. Harvey, "Asymptotically exact parabolic solutions of the generalized nonlinear Schrödinger equation with varying parameters," J. Opt. Soc. Am. B 23, 2541-2550 (2006).

6. C. Billet, J. M. Dudley, N. Joly, and J. C. Knight, "Intermediate asymptotic evolution and photonic bandgap fiber compression of optical similaritons around $1550 \mathrm{~nm}$," Opt. Express 13, 3236-3241 (2005).

7. C. Finot, G. Millot, S. Pitois, C. Billet, and J. M. Dudley, "Numerical and experimental study of parabolic pulses generated via Raman amplification in standard optical fibers," IEEE J. Sel. Top. Quantum Electron. 10, 1211-1218 (2004).

8. F. Parmigiani, C. Finot, K. Mukasa, M. Ibsen, M. A. F. Roelens, P. Petropoulos, and D. J. Richardson, "Ultra-flat SPM-broadened spectra in a highly nonlinear fiber using parabolic pulses formed in a fiber Bragg grating," Opt. Express 14, 7617-7622 (2006).

9. F. Ö. Ilday, J. Buckley, F. W. Wise, and W. G. Clark, "Self-similar evolution of parabolic pulses in a laser," Phys. Rev. Lett. 92, 213902(4) (2004).

10. B. G. Bale and S. Wabnitz, "Strong spectral filtering for a similariton mode-locked fiber laser," Opt. Lett, 35, 2466-2468 (2010). 
11. C. Aguergaray, D. Mechin, V. Kruglov, and J. D. Harvey, "Experimental realizatin of a mode-locked parabolic Raman fiber oscillator," Opt. Express, 18, 8680-8687 (2010).

12. C. Finot, S. Pitois, and G. Millot, "Regenerative 40-Gb/s wavelength converter based on similariton generation," Opt. Lett. 30, 1776-1778 (2005).

13. S. Boscolo and S. K. Turitsyn, "All-optical signal regeneration by temporal slicing of nonlinearly flattened optical waveform," IEEE Photon. Technol. Lett. 17, 1235-1237 (2005).

14. S. Boscolo, S. K. Turitsyn, and K. J. Blow, "Time domain all-optical signal processing at a RZ optical receiver," Opt. Express 13, 6217-6227 (2005).

15. F. Parmigiani, P. Petropoulos, M. Ibsen, and D. J. Richardson, "Pulse retiming based on XPM using parabolic pulses formed in a fiber Bragg grating," IEEE Photon. Technol. Lett. 18, 829-831 (2006).

16. T. Hirooka and M. Nakazawa, "All-optical 40-GHz Time-Domain Fourier Transformation using XPM with a dark parabolic pulse," IEEE Photon. Technol. Lett. 20, 1869-1871 (2008).

17. Y. Park, M. H. Asghari, T.-J. Ahn, and J. Azaña, "Transform-limited picosecond pulse shaping based on temporal coherence synthesization," Opt. Express 15, 9584-9599 (2007).

18. P. Petropoulos, M. Ibsen, A. D. Ellis, and D. J. Richardson, "Rectangular pulse generation based on pulse re-shaping using a superstructured fiber Bragg grating," IEEE J. Lightwave Technol. 19, 746-752 (2001).

19. F. Parmigiani, M. Ibsen, T. T. Ng, L. Provost, P. Petropoulos, and D. J. Richardson, "An efficient wavelength converter exploiting a grating based saw-tooth pulse shaper," IEEE Photon. Technol. Lett. 20, 1461-1463 (2008).

20. T. Hirooka and M. Nakazawa, "Parabolic pulse generation by use of a dispersiondecreasing fiber with normal group-velocity dispersion," Opt. Lett. 29, 498-500 (2004).

21. C. Finot, B. Barviau, G. Millot, A. Guryanov, A. Sysoliatin, and S. Wabnitz, "Parabolic 
pulse generation with active or passive dispersion decreasing optical fibers," Opt. Express 15, 15824-15835 (2007).

22. C. Finot, L. Provost, P. Petropoulos, and D. J. Richardson, "Parabolic pulse generation through passive nonlinear pulse re-shaping in a normally dispersive two segment fiber device," Opt. Express 15, 852-864, 2007.

23. S. Boscolo, A. I. Latkin, and S. K. Turitsyn, "Passive nonlinear pulse shaping in normally dispersive fiber systems," IEEE J. Quantum Electron. 44, 1196-1203 (2008).

24. H. Wang, A. I. Latkin, S. Boscolo, P. Harper, and S. K. Turitsyn, "Generation of triangular-shaped optical pulses in normally dispersive fibre," J. Opt. 12, 035205(5) (2010).

25. J. Li, B. E. Olsson, M. Karlsson, and P. A. Andrekson, "OTDM add-drop multiplexer based on XPM-induced wavelength shifting in highly nonlinear fiber," IEEE J. Lightwave Technol. 23, 2654-2661 (2005).

26. A. I. Latkin, S. Boscolo, R. S. Bhamber, and S. K. Turitsyn, "Doubling of optical signals using triangular pulses," J. Opt. Soc. Am. B 26, 1492-1496 (2009).

27. R. S. Bhamber, S. Boscolo, A. I. Latkin, and S. K. Turitsyn, "All-optical TDM to WDM signal conversion and partial regeneration using XPM with triangular pulses," in Proceedings of the 34th European Conference on Optical Communication, paper Th.1.B.2 (2008).

28. R. R. Alfano, The Supercontinuum Laser Source (Springer, 2006).

29. J. M. Dudley, G. Genty, and S. Coen, "Supercontinuum generation in photonic crystal fiber," Rev. Mod. Phys. 78, 1135-1184 (2006).

30. X. Gu, M. Kimmel, A. Shreenath, R. Trebino, J. M. Dudley, S. Coen, and R. S. Windeler, "Experimental studies of the coherence of microstructure-fiber supercontinuum," Opt. Express 11, 2697-2703 (2003).

31. K. L. Corwin, N. L. Newbury, J. M. Dudley, S. Coen, S. A. Diddams, K. Weber, and R. S. Windeler, "Fundamental noise limitations to supercontinuum generation in mi- 
crostructure fiber," Phys. Rev. Lett. 90, 113904 (2003).

32. K. Mori, H. Takara, S. Kawanishi, M. Saruwatari, and T. Morioka, "Flatly broadened supercontinuum spectrum generated in a dispersion decreasing fibre with convex dispersion profile," Electron. Lett. 33, 1806-1808 (1997).

33. G. Genty, S. Coen, and J. M. Dudley, "Fiber supercontinuum sources (Invited)," J. Opt. Soc. Am. B 24, 1771-1785 (2007).

34. K. Chow, Y. Takushima, C. Lin, C. Shu, and A. Bjarklev, "Flat super-continuum generation based on normal dispersion nonlinear photonic crystal fibre," Electron. Lett. 42, 989-991 (2006).

35. A. M. Heidt, A. Hartung, G. W. Bosman, P. Krok, E. G. Rohwer, H. Schwoerer, and H. Bartelt, "Coherent octave spanning near-infrared and visible supercontinuum generation in all-normal dispersion photonic crystal fibers," Opt. Express 19, 3775-3787 (2011).

36. L. E. Hooper, P. J. Mosley, A. C. Muir, W. J. Wadsworth, and J. C. Knight, "Coherent supercontinuum generation in photonic crystal fiber with all-normal group velocity dispersion," Opt. Express 19, 4902-4907 (2011).

37. M. L. V. Tse, P. Horak, F. Poletti, N. G. R. Broderick, J. H. V. Price, J. R. Hayes, and D. J. Richardson, "Supercontinuum generation at $1.06 \mu \mathrm{m}$ in holey fibers with dispersion flattened profiles," Opt. Express 14, 4445-4451 (2006).

38. A. Hartung, A. M. Heidt, and H. Bartelt, "Design of all-normal dispersion microstructured optical fibers for pulse-preserving supercontinuum generation," Opt. Express 14, 7742-7749 (2011).

39. B. G. Bale and S. Boscolo, "Impact of third-order fibre dispersion on the evolution of parabolic optical pulses," J. Opt. 12, 015202(6) (2010).

40. G. P. Agrawal, Nonlinear Fiber Optics (3rd ed., Academic Press, 2001).

41. J. D. Harvey, R. Leonhardt, S. Coen, G. K. L. Wong, J. C. Knight, W. J. Wadsworth, and J. S. Russell, "Scalar modulation instability in the normal dispersion regime by use of a photonic crystal fiber," Opt. Lett. 28, 2225-2227 (2003). 
42. K. Hammani, C. Finot, B. Kibler, and G. Millot, "Soliton generation in a microstructured fiber by fourth-order scalar modulation instability," IEEE Photon. J. 1, 205-212 (2009).

43. E. R. Andresen, J. M. Dudley, D. Oron, C. Finot, and H. Rigneault, "Transform-limited spectral compression by self-phase modulation of amplitude shaped pulses with negative chirp," Opt. Lett. 36, 707-709 (2011).

44. G. B. Whitham, Linear and Nonlinear Waves (1st ed., Wiley Interscience,1974).

45. A. Ruehl, O. Prochnow, D. Wandt, D. Kracht, B. Burgoyne, N. Godbout, and S. Lacroix, "Dynamics of parabolic pulses in an ultrafast fiber laser," Opt. Lett. 31, 2734-2736 (2006).

46. C. Finot, B. Kibler, L. Provost, and S. Wabnitz, "Beneficial impact of wave-breaking or coherent continuum formation in normally dispersive nonlinear fibers," J. Opt. Soc. Am. B 25, 1938-1948 (2008). 


\section{List of Figure Captions}

Fig. 1 Convex dispersion profile for PCF under consideration. Pumping occurs at the extremum of dispersion. This profile is similar to that experimentally realized in Ref. [37].

Fig. 2 Characteristic curves (7a) for $\left|\Omega_{0}\right| \sim 2 N^{2} \xi_{0}=400$ and $\delta \Omega_{0}^{2} / 6=0.5$ (solid) and $\delta=0$ (dashed). These values correspond to reasonable values used in simulations of $N^{2} \sim 2 \times 10^{5}$ and $\delta \sim 2 \times 10^{-5}$. The characteristic curves plotted are for $\left|\tau_{0}\right|=0$ (black), 1 (blue), 2 (red) and 3 (green).

Fig. 3 Power reduction factor Eq. (8) for $\Omega_{0} \sim-2 N^{2} \xi_{0}=-400, \delta \Omega_{0}^{2} / 6=0.5$, and $\left|\tau_{0}\right|=0$ (black), 0.5 (cyan), 1 (blue), 2 (red) and 3 (green).

Fig. 4 Temporal power profiles (a) without and (b) with FOD at propagation distances $\xi=0$ (red), $\xi=0.0011$ (green), $\xi=0.0022$ (blue), $\xi=0.0033$ (cyan), and $\xi=0.0041$ (magenta). The parameters are $N^{2}=2.6474 \times 10^{5}$, (a) $\delta=0$ and (b) $\delta=2.3669 \times 10^{-5}$. The inset shown in (b) is the temporal profile (black) at $\xi=0.0028$, and its fit to a triangular function (red circles).

Fig. 5 Spectral power profiles (a) without and (b) with FOD at propagation distances $\xi=0$ (red), $\xi=0.0011$ (green), $\xi=0.0022$ (blue), $\xi=0.0033$ (cyan), and $\xi=0.0041$ (magenta). The parameters are the same as in Fig. 4 and $\omega$ is normalized frequency.

Fig. 6 Longitudinal evolutions of (a) The misfit parameter to a parabolic temporal shape with no FOD (dashed) and a triangular temporal shape with FOD (solid) and (b) the RMS spectral bandwidth without (dashed) and with (solid) FOD. The parameters are $\delta=0$ or $\delta=2.3669 \times 10^{-5}$ and $N^{2}=2.6474 \times 10^{5}$.

Fig. 7 Longitudinal evolutions of the misfit parameter to a triangular temporal shape for varying values of (a) $\delta$ and (b) $N^{2} . S=S^{*} / 2$ (blue), $2 S^{*} / 3$ (cyan), $S^{*}$ (dashed), $3 S^{*} / 2$ (red), $2 S^{*}$ (black), with (a) $S^{*}=\delta^{*}=2.3669 \times 10^{-5}$ and $N^{2}=2.6474 \times 10^{5}$ and (b) $S^{*}=N^{* 2}=2.6474 \times 10^{5}$ and $\delta=2.3669 \times 10^{-5}$.

Fig. 8 (a) Temporal and (b) spectral intensity profiles for $N^{2}=N^{* 2} / 2, N^{* 2}, 2 N^{* 2}$, with $N^{* 2}=2.6474 \times 10^{5}$. For $N^{2}=2 N^{* 2}$ a best fit triangular temporal shape is also shown 
(dashed). The temporal profiles shown are taken at the circled points of Fig. 7(b), and the spectral profiles are shown at points of maximum flatness. The parameter $\delta=2.3669 \times 10^{-5}$. Fig. 9 Spectro-temporal plots at propagation distance $\xi=0.0013$ for initial chirp-free (a) parabolic and (b) hyperbolic secant intensity profiles with the same FWHM pulse duration and energy. Note that the parabolic initial condition keeps a monotonic chirp across the pulse profile, where the initial hyperbolic secant pulse does not.

Fig. 10 (a) Temporal and (b) spectral powers at $\xi=0.0013$ for parabolic (black), Gaussian (green), and hyperbolic secant (red) initial conditions. Note that the high oscillations induced for the initial Gaussian and hyperbolic secant pulses. 


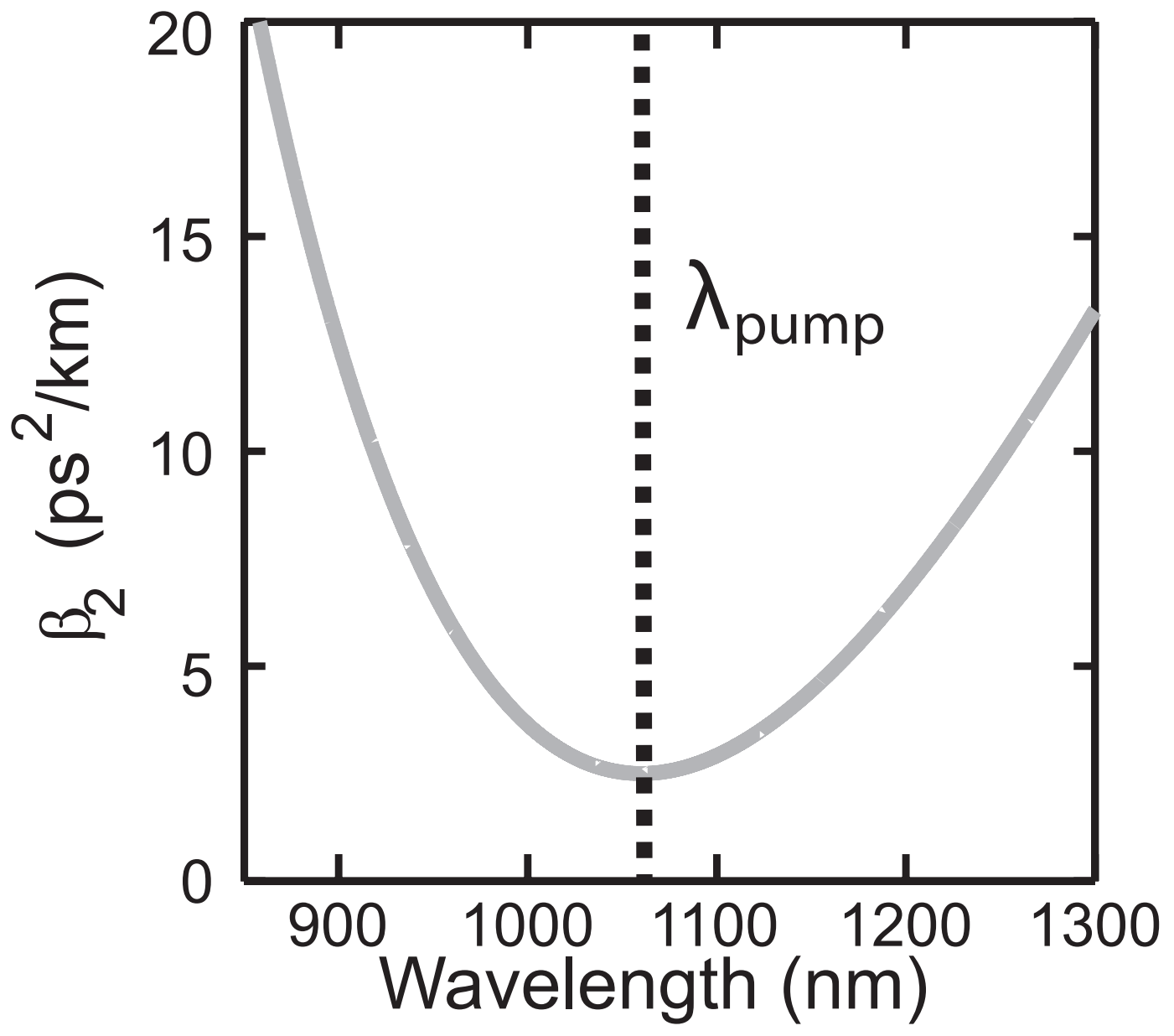

Fig. 1. Convex dispersion profile for PCF under consideration. Pumping occurs at the extremum of dispersion. This profile is similar to that experimentally realized in Ref. [37]. 


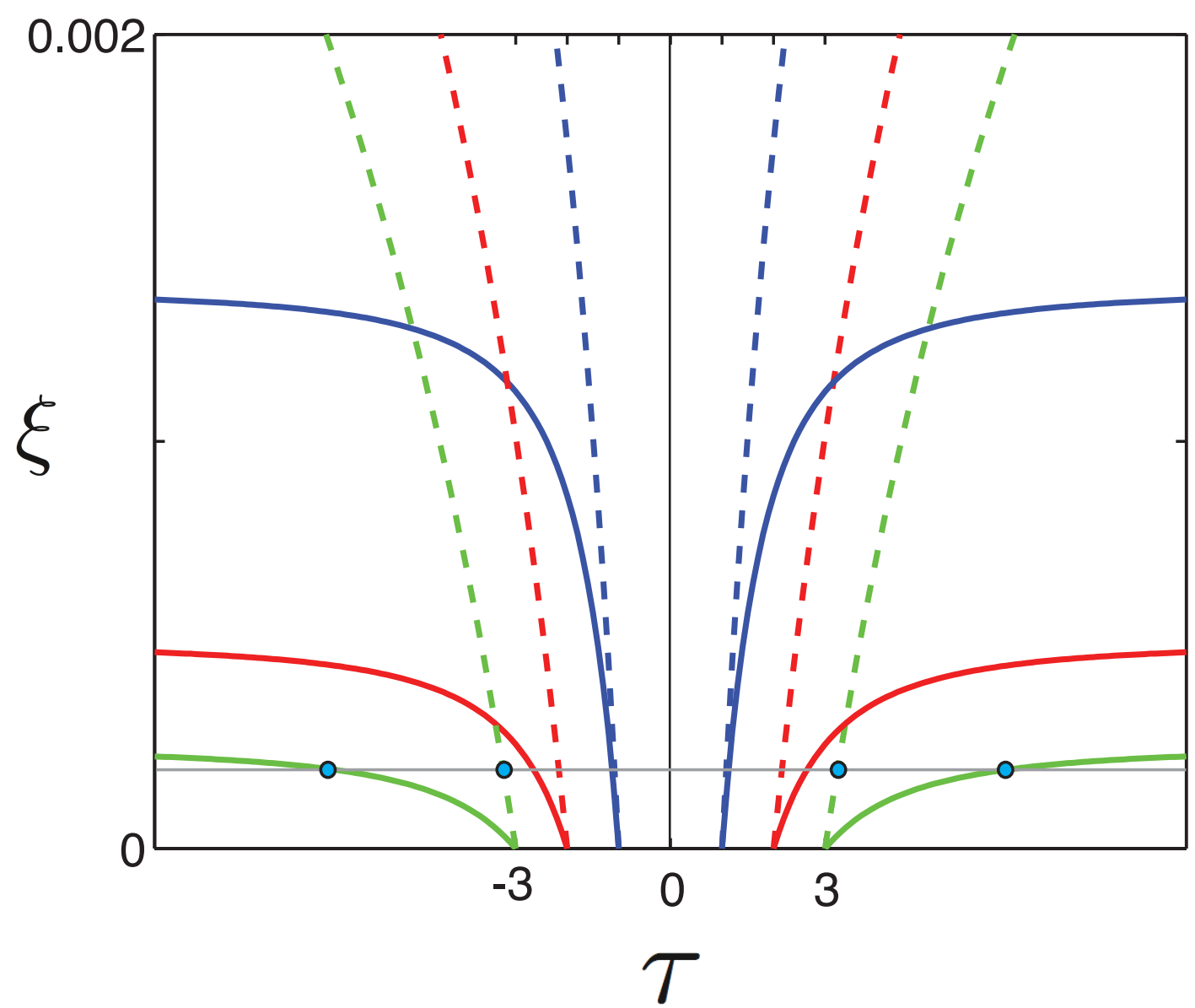

Fig. 2. Characteristic curves (7a) for $\left|\Omega_{0}\right| \sim 2 N^{2} \xi_{0}=400$ and $\delta \Omega_{0}^{2} / 6=0.5$ (solid) and $\delta=0$ (dashed). These values correspond to reasonable values used in simulations of $N^{2} \sim 2 \times 10^{5}$ and $\delta \sim 2 \times 10^{-5}$. The characteristic curves plotted are for $\left|\tau_{0}\right|=0$ (black), 1 (blue), 2 (red) and 3 (green). 


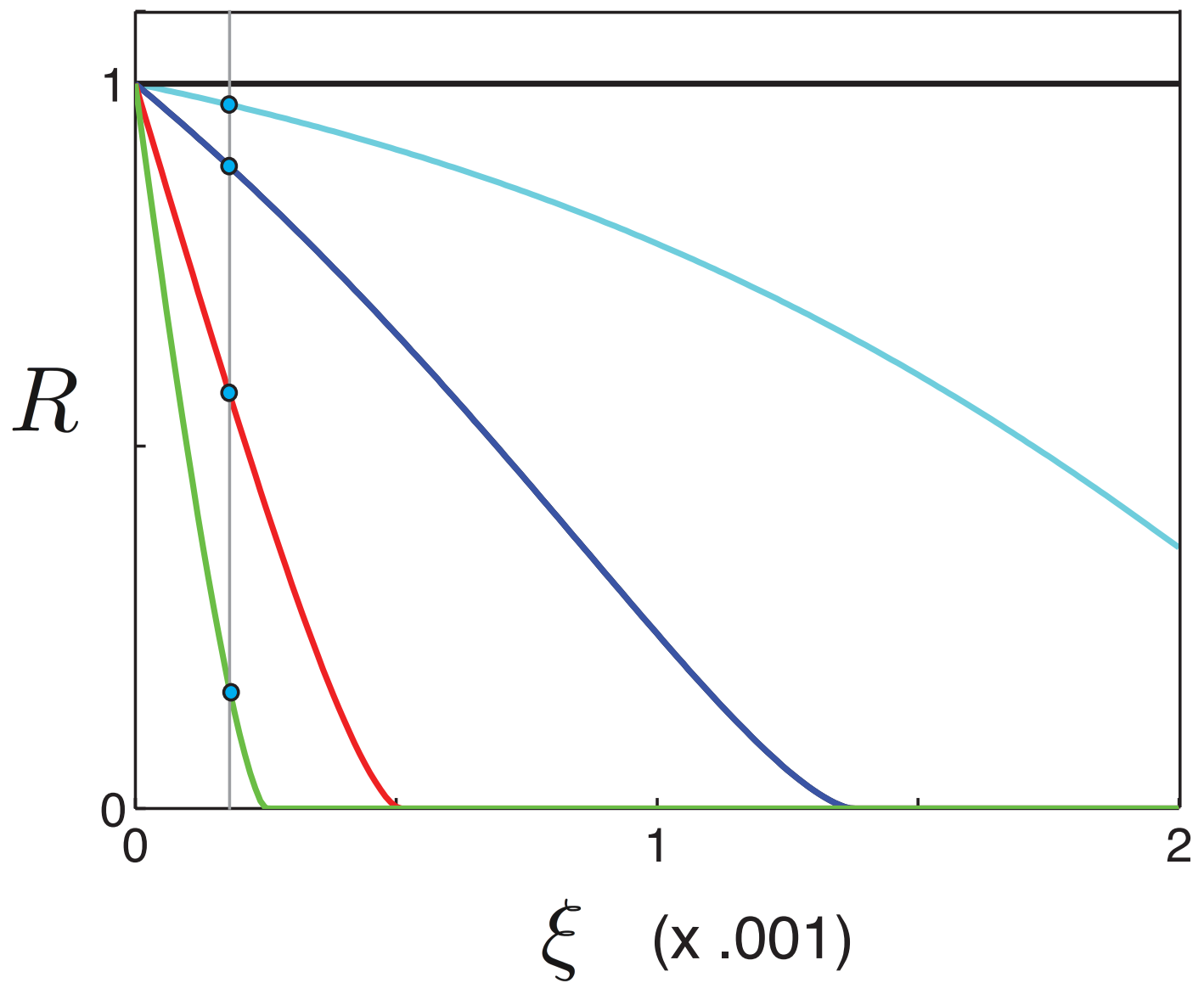

Fig. 3. Power reduction factor Eq. (8) for $\Omega_{0} \sim-2 N^{2} \xi_{0}=-400, \delta \Omega_{0}^{2} / 6=0.5$, and $\left|\tau_{0}\right|=0$ (black), 0.5 (cyan), 1 (blue), 2 (red) and 3 (green). 

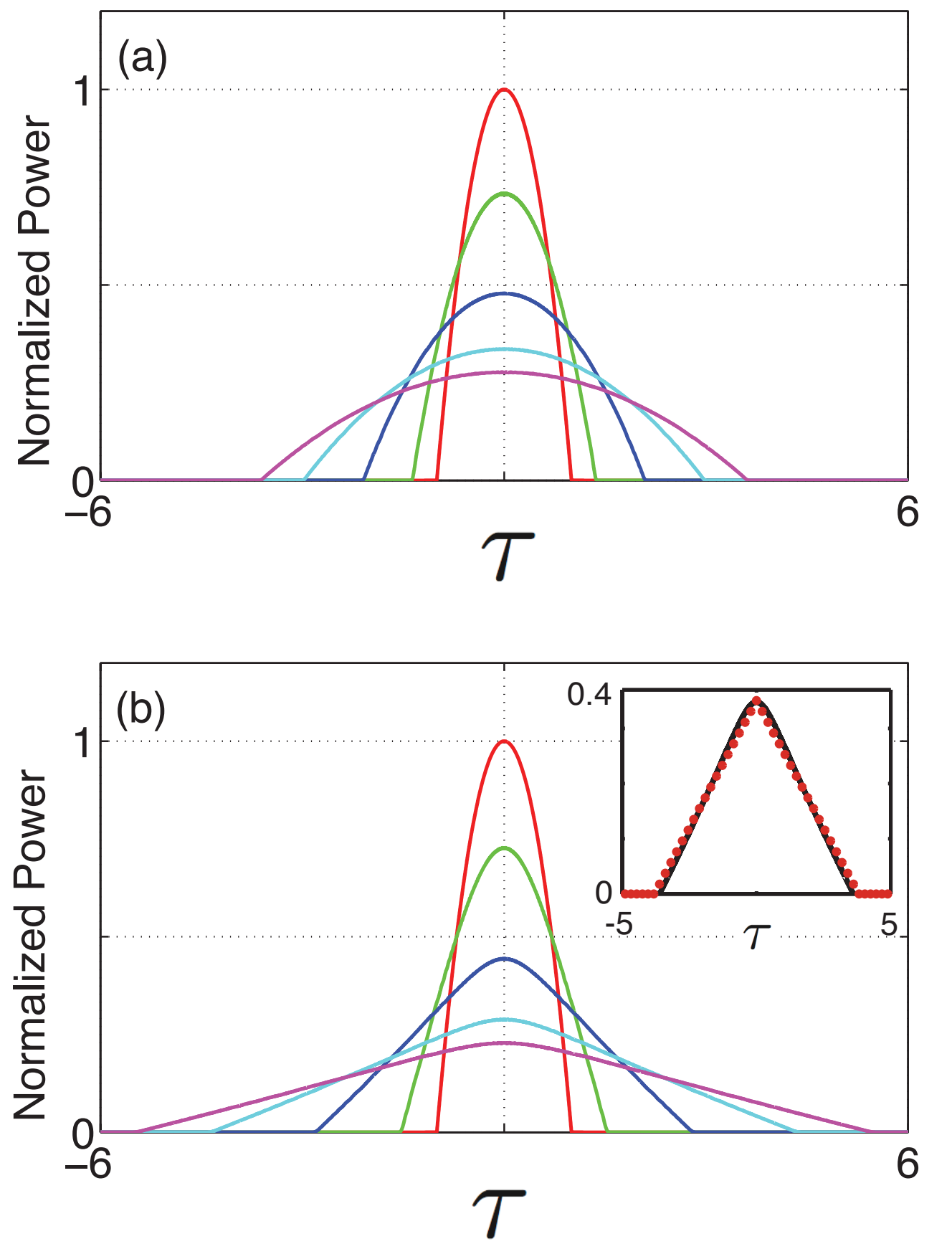

Fig. 4. Temporal power profiles (a) without and (b) with FOD at propagation distances $\xi=0$ (red), $\xi=0.0011$ (green), $\xi=0.0022$ (blue), $\xi=0.0033$ (cyan), and $\xi=0.0041$ (magenta). The parameters are $N^{2}=2.6474 \times 10^{5}$, (a) $\delta=0$ and (b) $\delta=2.3669 \times 10^{-5}$. The inset shown in (b) is the temporal profile (black) at $\xi=0.0028$, and its fit to a triangular function (red circles). 

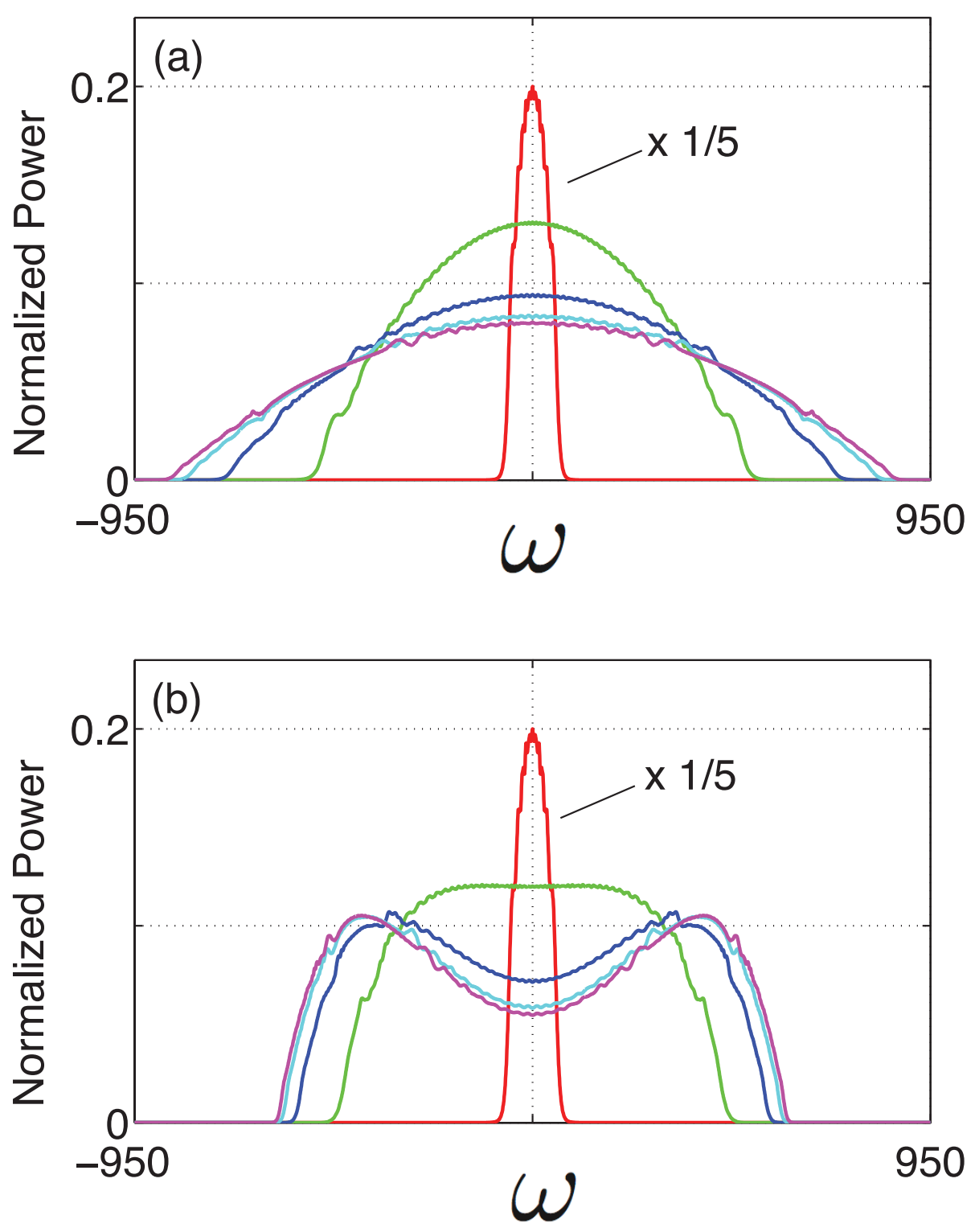

Fig. 5. Spectral power profiles (a) without and (b) with FOD at propagation distances $\xi=0$ (red), $\xi=0.0011$ (green), $\xi=0.0022$ (blue), $\xi=0.0033$ (cyan), and $\xi=0.0041$ (magenta). The parameters are the same as in Fig. 4 and $\omega$ is normalized frequency. 

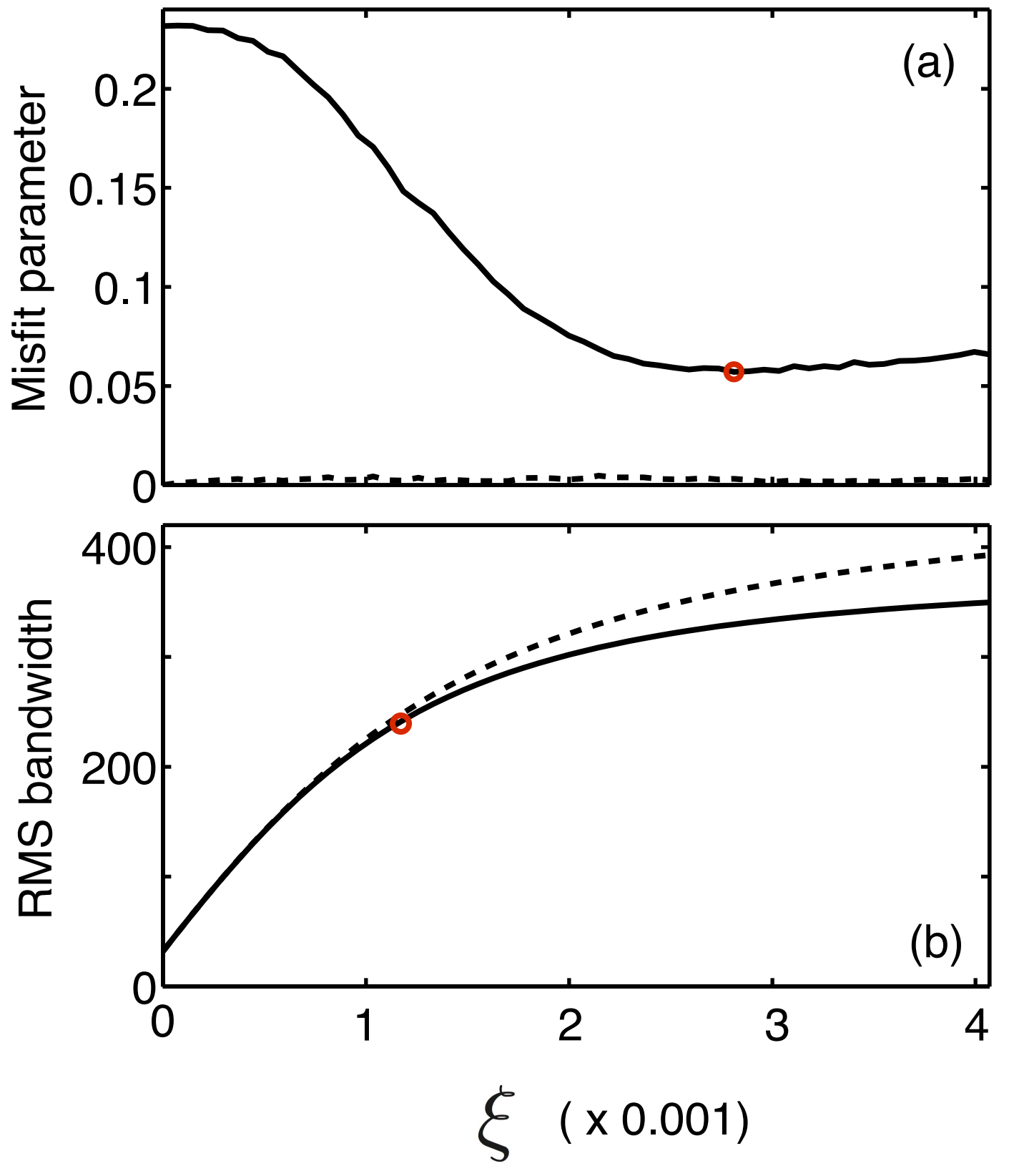

Fig. 6. Longitudinal evolutions of (a) The misfit parameter to a parabolic temporal shape with no FOD (dashed) and a triangular temporal shape with FOD (solid) and (b) the RMS spectral bandwidth without (dashed) and with (solid) FOD. The parameters are $\delta=0$ or $\delta=2.3669 \times 10^{-5}$ and $N^{2}=2.6474 \times 10^{5}$. 

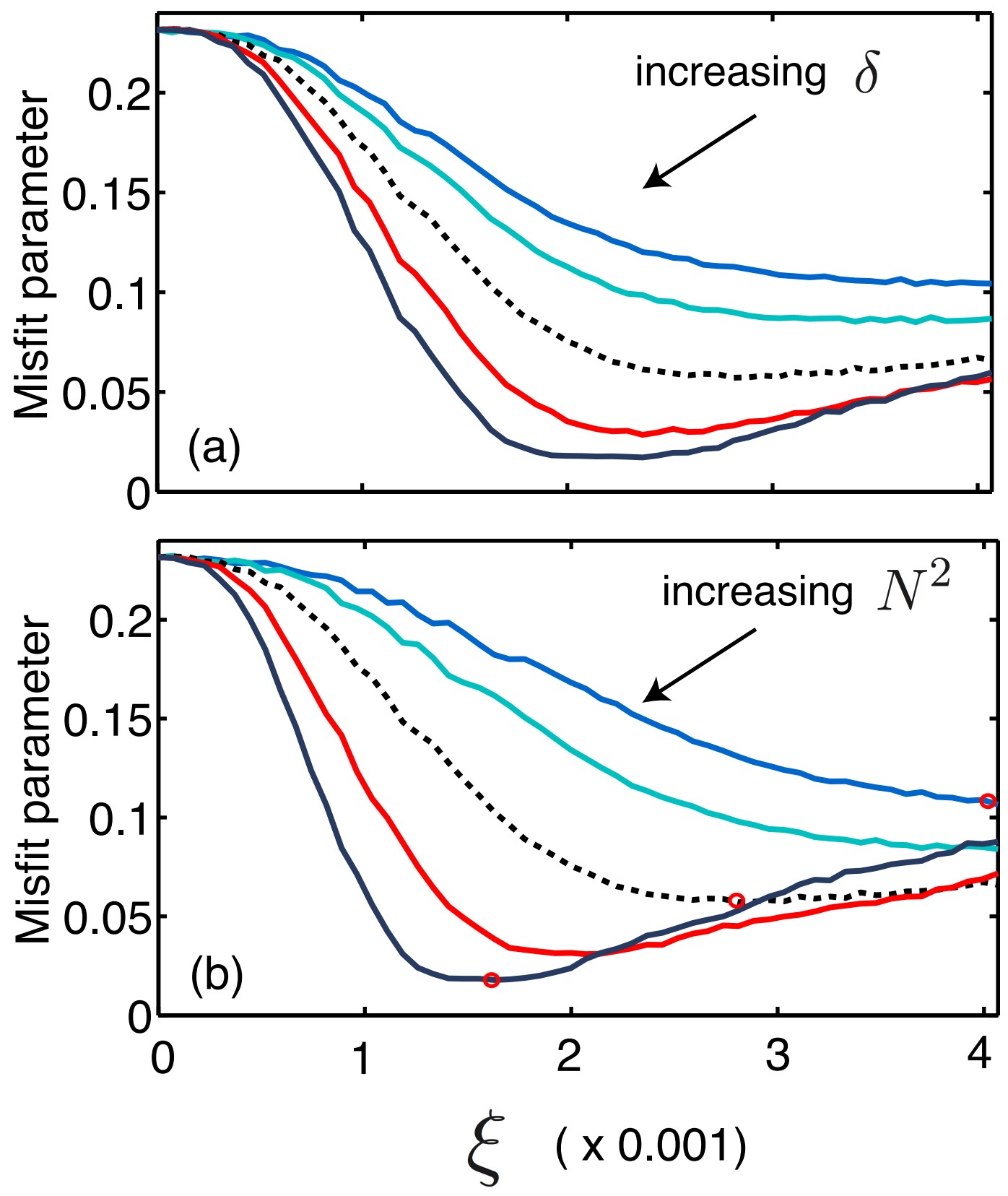

Fig. 7. Longitudinal evolutions of the misfit parameter to a triangular temporal shape for varying values of (a) $\delta$ and (b) $N^{2} . S=S^{*} / 2$ (blue), $2 S^{*} / 3$ (cyan), $S^{*}$ (dashed), $3 S^{*} / 2$ (red), $2 S^{*}$ (black), with (a) $S^{*}=\delta^{*}=2.3669 \times 10^{-5}$ and $N^{2}=2.6474 \times 10^{5}$ and (b) $S^{*}=N^{* 2}=2.6474 \times 10^{5}$ and $\delta=2.3669 \times 10^{-5}$. 

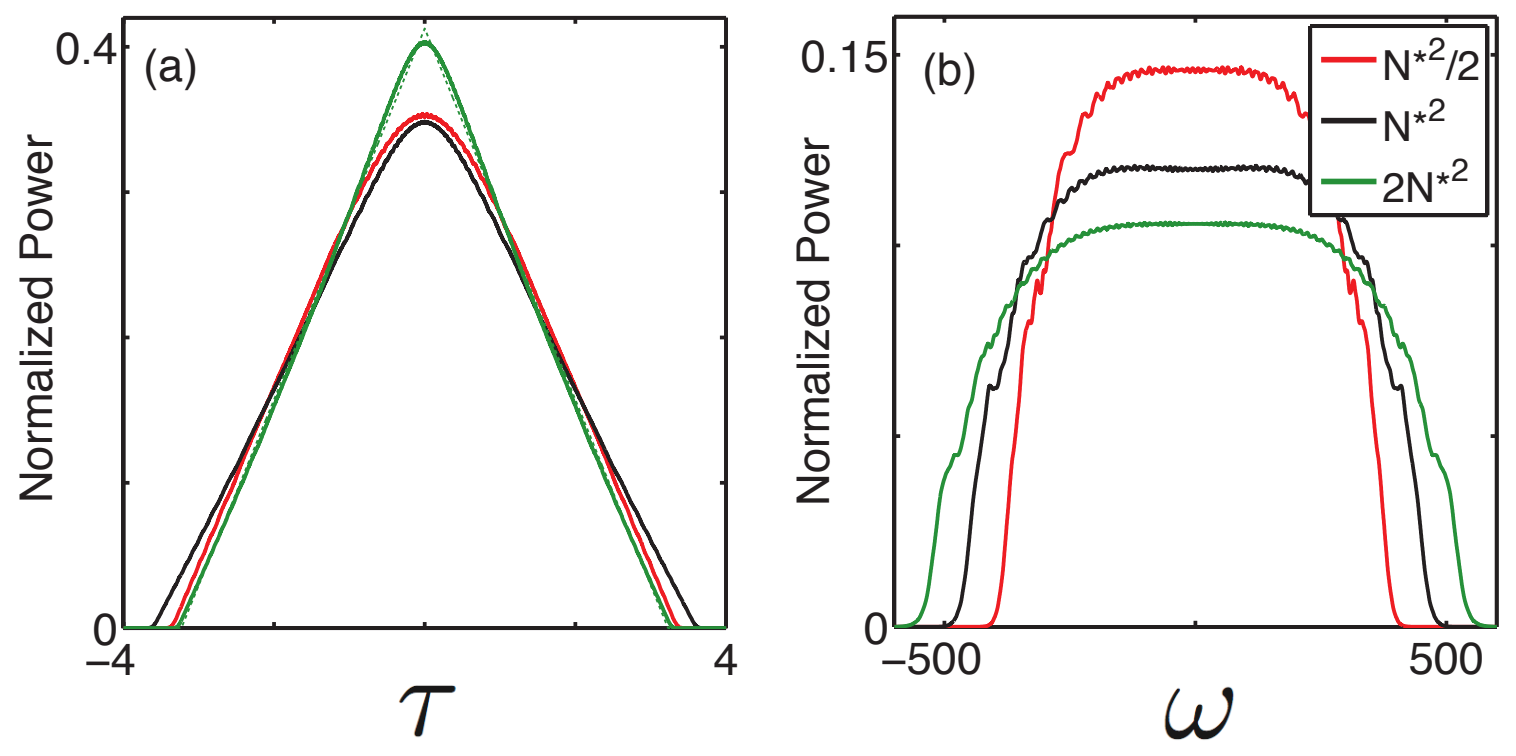

Fig. 8. (a) Temporal and (b) spectral intensity profiles for $N^{2}=N^{* 2} / 2, N^{* 2}, 2 N^{* 2}$, with $N^{* 2}=2.6474 \times 10^{5}$. For $N^{2}=2 N^{* 2}$ a best fit triangular temporal shape is also shown (dashed). The temporal profiles shown are taken at the circled points of Fig. $7(\mathrm{~b})$, and the spectral profiles are shown at points of maximum flatness. The parameter $\delta=2.3669 \times 10^{-5}$. 


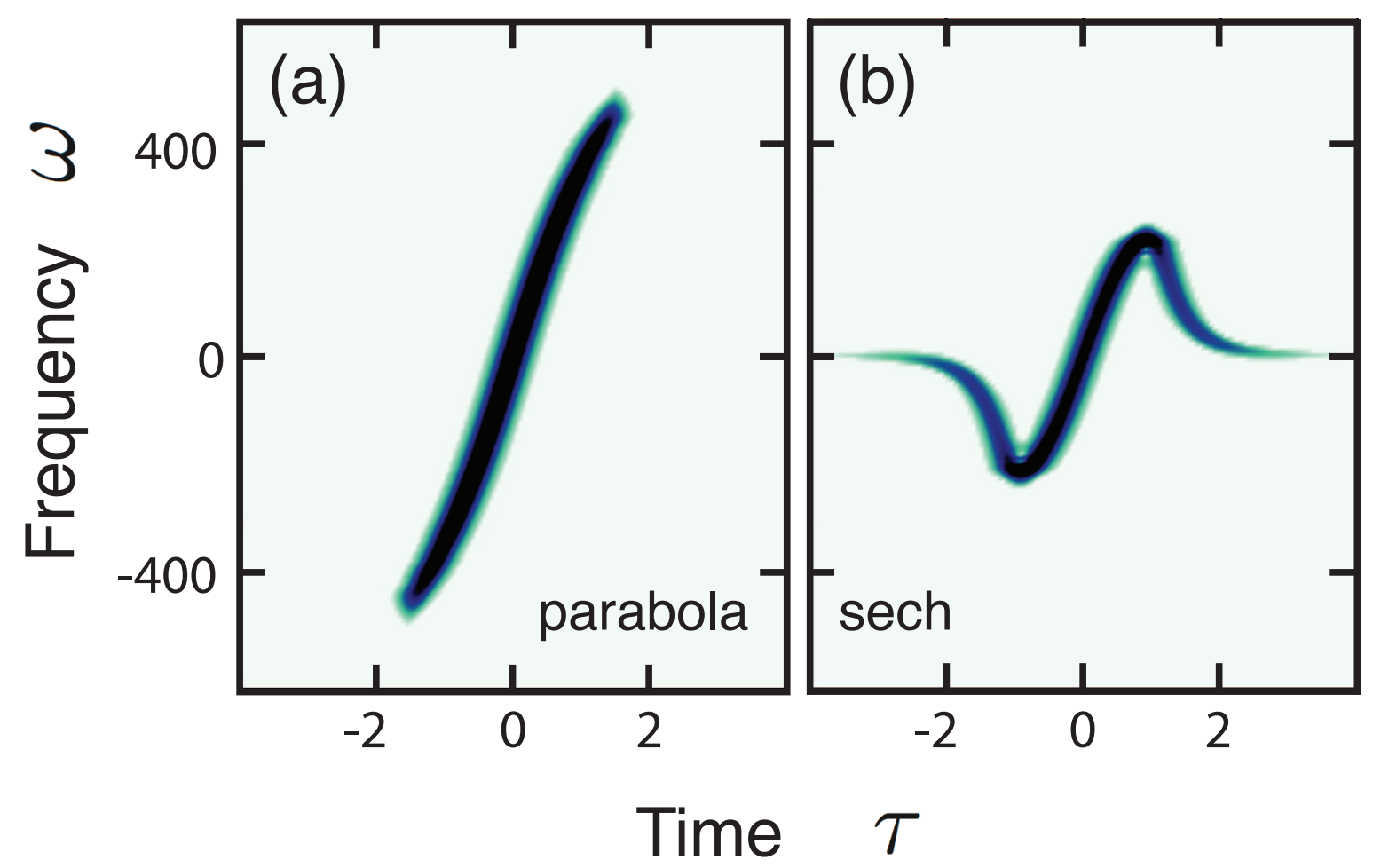

Fig. 9. Spectro-temporal plots at propagation distance $\xi=0.0013$ for initial chirp-free (a) parabolic and (b) hyperbolic secant intensity profiles with the same FWHM pulse duration and energy. Note that the parabolic initial condition keeps a monotonic chirp across the pulse profile, where the initial hyperbolic secant pulse does not. 

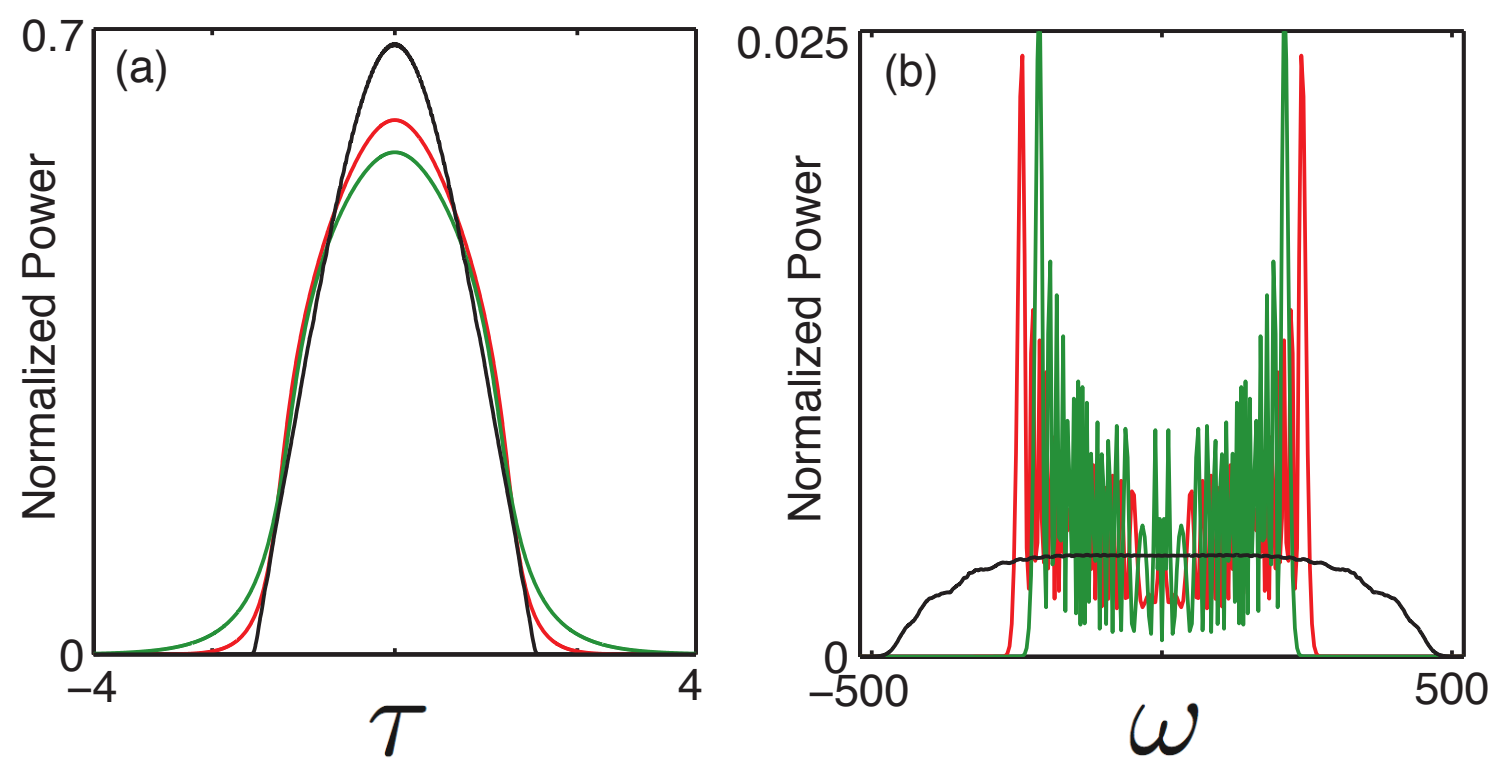

Fig. 10. (a) Temporal and (b) spectral powers at $\xi=0.0013$ for parabolic (black), Gaussian (green), and hyperbolic secant (red) initial conditions. Note that the high oscillations induced for the initial Gaussian and hyperbolic secant pulses. 